RESEARCH REPORT

\title{
Employee worktime control moderates the effects of job strain and effort-reward imbalance on sickness absence: the 10-town study
}

\author{
Leena Ala-Mursula, Jussi Vahtera, Anne Linna, Jaana Pentti, Mika Kivimäki
}

J Epidemiol Community Health 2005;59:851-857. doi: 10.1136/jech.2004.030924

\begin{abstract}
Study objective: To examine whether the effects of work stress on sickness absence vary by the level of control the employees have over their working times.

Design: Prospective cohort study. A survey of job strain, effort-reward imbalance, and control over daily working hours and days off was carried out in 2000-01. The survey responses were linked with registered data on the number of medically certified ( $>3$ days) sickness absences from one year before the survey until the end of 2003. The mean follow up period was 28.2 (SD 8.1) months. Adjustments were made for demographics and behavioural health risks. Aggregated measures of worktime control according to workplaces were used to control for differences in reactivity and response style.

Setting: Ten towns in Finland. Participants: 16139 public sector employees who had no medically certified sickness absences in the year preceding the survey.

Main results: Among the women, individually measured control over daily working hours and days off moderated the association between work stress and sickness absence. The combination of high stress and good worktime control was associated with lower absence rates than a combination of high stress and poor worktime control. This finding was replicated in the analyses using workplace aggregates of worktime control. Among the men, the findings were less consistent and not replicable using aggregated measures of worktime control.

Conclusions: Good control over working times reduces the adverse effect of work stress on sickness absence especially among female employees.
\end{abstract}

See end of article for authors' affiliations

Correspondence to: $\operatorname{Dr} L$ Ala-Mursula, Centre of Occupational Health, POBox 36, FIN-90015 Oulu, Finland; Leena.AlaMursula@oulu.fi

Accepted for publication 22 April 2005

W ork stress has been shown to affect several public health domains such as perceived health, ${ }^{12}$ psychological health, ${ }^{3}$ cardiovascular morbidity, ${ }^{4}$ sickness absence, ${ }^{6-8}$ and mortality. ${ }^{10}{ }^{11}$ In specifying the backgrounds of these health effects, the two most prevalent work stress models have identified health risks in "job strain" resulting from a combination of high job demands with low control over job content ${ }^{12}$ and in "effort-reward imbalance" originating from a lack of reciprocity between high efforts spent at work with low rewards received. ${ }^{13}$

There is an urgent need for evidence on tools that could alleviate the health impairments related to work stress. ${ }^{14} \mathrm{~A}$ potential protecting factor could be employee control over working times, recently shown to predict health especially among female employees. ${ }^{15} 16$ The health benefits of worktime control may stem from advantages in combining work and non-work demands. Worktime control could also be used to flee from particularly stressful work situations and to choose working at times of best possible resources and support.

Previously, European Union survey analyses have suggested that working time autonomy helps to reduce the health risk relating to unfavourable working times, but that the positive effect is weak compared with the negative effect of unsociable hours. ${ }^{17}$ A nationwide US survey has implied that scheduling control could benefit all workers, not just those with conflicting demands, and regardless of schedule. ${ }^{18}$ A Swedish study using occupational level aggregates has suggested that low influence on working hours could be more deleterious to cardiovascular health in hectic high strain jobs than in other job strain situations. ${ }^{19}$
A limitation of all the above studies is that they have not directly tested whether higher worktime control could reduce the adverse effects of work stress. In this study, we examined whether work stress more strongly predicts health problems among employees with poor control over working times than among those with good worktime control, in a large cohort of full time employees covering a variety of occupations. We defined work stress as suggested in the job strain model and in the effort-reward imbalance model, and linked the survey responses to data on medically certified sickness absence from the employers' records, a reliable global measure of health in working populations..$^{20-22}$

\section{METHODS}

\section{Design and participants}

Data were drawn from the ongoing 10-town study exploring employee health in 10-towns in Finland. In 2000 and 2001, 32299 identifiable full time employees responded to a questionnaire assessing worktime control, job control, job demands, job efforts and rewards, and health behaviour. The response rate was $67 \%$. Of the respondents, we selected the 16139 respondents who had no medically certified sickness absences in the year preceding the survey and who had more than six months of follow up. Their health problems in the years after the survey, as indicated by sickness absence, were monitored until the end of 2003. The mean follow up time was 28.2 (SD 8.1) months.

The sample did not substantially differ from the eligible population (that is, all the 27145 permanent or long term employees contracted for more than nine months in the survey year and for more than six months during the follow up) in terms of age, occupational status, and sickness 
absence. In the sample, the mean age was 44 years, the proportion of upper white collar workers $41 \%$, lower white collar workers $43 \%$, and manual workers $16 \%$, and the rate of medically certified sickness absence 0.50 per person year. In the eligible population, the mean age was 44 years, $40 \%$ were upper white collar workers, $41 \%$ lower white collar workers, and $19 \%$ manual workers, and the absence rate was 0.53 . The proportions of women (75\%) and permanent employees $(82 \%)$ were somewhat higher in the sample than in the eligible population ( $70 \%$ women, $78 \%$ permanent). The ethics committee of the Finnish Institute of Occupational Health approved the study.

\section{Worktime control}

The respondents rated on a $1-5$ scale (very little to very much) how much they were able to influence the following aspects of their working times: length of a workday, the starting and ending times of a workday, the taking of breaks during the workday, the handling of private matters during the workday, the scheduling of work shifts, the scheduling of vacations and paid days off, and the taking of unpaid leave. These variables were then considered as separate measures in a factor analysis using principal component analysis with varimax rotation and eigenvalue criterion $>1$ for factor retention. Two factors accounted for $81.7 \%$ of the total variance. The first factor "control over daily working hours" (55.5\% of the total variance, Cronbach's $\alpha 0.86$, mean 2.31 , SD 1.2) consisted of control over the length of a workday and the starting and ending times of a workday, and the second factor "control over days off" $(26.2 \%$ of the total variance, Cronbach's $\alpha$ 0.67, mean 3.18, SD 1.0) of influencing the taking and timing of vacations and other paid and unpaid leave. Their mutual Pearson correlation coefficient was 0.35 . The correlation coefficients with job control (below) were 0.24 for control over daily working hours and 0.09 for control over days off and the corresponding figures with decision authority (a subscale of job control) were 0.27 and 0.18 , respectively. The correlation coefficients with effort-reward imbalance (below) were -0.11 for both worktime control factors.

\section{Work stress}

The measures of the job strain model were derived from the Job Content Questionnaire. ${ }^{23}$ The job demands scale dealt with workload and work pace (five items; Cronbach's $\alpha 0.81$; range $1-5$; mean 3.27 and SD 0.8 ) and the job control scale concerned decision authority and skill discretion (eight items; Cronbach's $\alpha$ 0.82; range $1-5$; mean 3.90 and SD $0.7)$. The means of the aforementioned sum scores were further divided into quartiles. Job strain situations were cross tabulated from the median splits of job control and job demands, and categorised as low strain (low demands with high control), active jobs (high demands with high control), passive jobs (low demands with low control), and high strain (high demands with low control). ${ }^{12}$

We measured the employees' effort at work with a single question "How much do you feel you invest in your job in terms of skill and energy?"; (range 1-5, mean 4.24, SD 0.7) and rewards from work by three items capturing how much the respondents feel they get in return from work in terms of income and job benefits, recognition and prestige, and personal satisfaction (Cronbach's $\alpha$ 0.64; range $1-5$, mean 1.54, SD 0.5). ${ }^{24}{ }^{25} \mathrm{~A}$ measure of effort-reward imbalance was then obtained by dividing effort by rewards and categorising the resulting quotient into quartiles.

\section{Sickness absences}

We used information on medically certified sickness absences from the employers' registers to measure health. ${ }^{20-22}$ The study cohort was selected on the basis of having no such absences in the year preceding the survey, either 1999 or 2000. Health prospects were monitored through sickness absences in the years after the survey until the end of 2003. Absences in the survey year were not noted. All sick leaves are reliably recorded, including the dates when each period started and ended. Medical certificates are required for sick leaves longer than three days, the focus of this study. The employees are paid full salary during sick leave. Maternity leaves and absences to care for a sick child are not recorded as sick leave. The regulations permit up to three paid days off work to care for acutely ill children under 10 years of age and the annual number of such three day periods is not limited. Thus the participants had no reason to falsely report being ill when caring for a sick child.

\section{Background variables}

Information on age, sex, type of work contract (permanent/ fixed term), ${ }^{26}$ and occupational status were obtained from the employers' records. The occupational titles, expressed as fivedigit codes of Statistics Finland, were categorised into the occupational statuses of manual and lower and upper white collar work. ${ }^{27}$ The survey responses regarding the level of education were categorised as no vocational education, vocational education, college level, and university education. The responses on work schedule were classified into standard hours (weekdays, daytime only), evening and/or weekend shifts but no night shifts, and also night shifts.. ${ }^{28}$ Of the family characteristics, we surveyed marital status (married/ cohabiting versus others) and family type: no children, only preschool child(ren) 0-6 years of age, only schoolchild(ren) 7-18 years of age, or children in both age groups. The behavioural and biological health risks measured were smoking status (current smokers or non-smokers), high alcohol consumption ( $>200$ grams of alcohol per week), sedentary lifestyle ( $<30$ minutes of fast walking per week), and overweight (body mass index $>27 \mathrm{~kg} / \mathrm{m}^{2}$ ).

\section{Statistical analyses}

We used analyses of variance to study the relations of control over daily working hours and control over days off with background variables. After checking the employers' sickness absence records and combining the overlapping and consecutive periods, we calculated the individual numbers of sick leave periods and the individual person years representing "days at risk for sickness absence" by excluding the days absent for reasons other than sickness.

The effects of worktime control and work stress on the rates of sick leaves per person years were studied by Poisson regression models and expressed as rate ratios (RR) and their 95\% confidence intervals (95\% CI). Use of the Poisson model implies that the between-employee variance in the rates of sick leave equals the expected rate of sick leave. In this study, the dispersion of medically certified sick leaves did not significantly deviate from the model's assumptions.

To study the joint effects of worktime control and work stress on sickness absence, we formulated combination variables by cross tabulating the measures of work stress (in quartiles) and the levels of worktime control (median splits). The effects of the resulting eight combinations of work stress with worktime control on subsequent sickness absence were studied by Poisson regression models using the hypothetically least adverse condition (lowest quartile of work stress with good worktime control) as the reference category. The significance of these joint effects was studied using appropriate cross product terms (work stress $\times$ worktime control).

To reduce bias arising from differences in response styles and reactivity, we replicated the analyses using aggregated 
Table 1 Relations of background variables with measures of worktime control

\begin{tabular}{|c|c|c|c|c|c|c|}
\hline & & \multirow[b]{2}{*}{ Number (\%) } & \multicolumn{2}{|c|}{ Control over daily working hours* } & \multicolumn{2}{|c|}{ Control over days offt } \\
\hline & & & mean (SD) & p valueł & mean (SD) & p value \\
\hline \multirow[t]{2}{*}{ Gender } & Men & $4012(25)$ & $2.41(1.3)$ & $<0.001$ & $3.19(1.1)$ & 0.354 \\
\hline & Women & $12127(75)$ & $2.27(1.1)$ & & $3.17(1.0)$ & \\
\hline \multirow[t]{3}{*}{ Age group } & $18-39$ & $4967(31)$ & $2.30(1.1)$ & 0.006 & $3.12(1.0)$ & $<0.001$ \\
\hline & $40-49$ & $5650(35)$ & $2.34(1.2)$ & & $3.28(1.0)$ & \\
\hline & $50-64$ & $5522(34)$ & $2.27(1.2)$ & & $3.13(1.1)$ & \\
\hline \multirow[t]{2}{*}{ Marital status } & Married or cohabiting & $12298(77)$ & $2.31(1.2)$ & 0.373 & $3.18(1.0)$ & 0.791 \\
\hline & Single, separated, widowed & $3689(23)$ & $2.29(1.2)$ & & $3.18(1.0)$ & \\
\hline \multirow[t]{4}{*}{ Family type } & No children & $7378(46)$ & $2.31(1.2)$ & 0.002 & $3.17(1.0)$ & $<0.001$ \\
\hline & Child(ren) 0-6 years & 2149 (13) & $2.22(1.2)$ & & $3.12(1.0)$ & \\
\hline & Child(ren) 7-18 years & $4789(30)$ & $2.34(1.2)$ & & $3.24(1.0)$ & \\
\hline & Children $0-18$ years & $1682(11)$ & $2.29(1.1)$ & & $3.16(1.0)$ & \\
\hline \multirow{4}{*}{$\begin{array}{l}\text { Level of } \\
\text { education }\end{array}$} & University & 4953 (32) & $2.57(1.2)$ & $<0.001$ & $2.82(1.1)$ & $<0.001$ \\
\hline & College & $5800(37)$ & $2.46(1.2)$ & & $3.44(0.9)$ & \\
\hline & Vocational & 2964 (19) & $1.88(1.0)$ & & $3.27(0.9)$ & \\
\hline & None & 1879 (12) & $1.88(1.0)$ & & $3.17(1.0)$ & \\
\hline \multirow{3}{*}{$\begin{array}{l}\text { Occupational } \\
\text { status }\end{array}$} & Upper white collar & $6577(42)$ & $2.54(1.2)$ & $<0.001$ & $2.92(1.1)$ & $<0.001$ \\
\hline & Lower white collar & $6797(43)$ & $2.27(1.2)$ & & $3.46(0.9)$ & \\
\hline & Manual worker & $2468(16)$ & $1.76(1.0)$ & & $3.11(1.0)$ & \\
\hline \multirow{5}{*}{$\begin{array}{l}\text { Permanent } \\
\text { employment } \\
\text { Schedule type }\end{array}$} & Yes & $13164(82)$ & $2.29(1.2)$ & $<0.001$ & $3.24(1.0)$ & $<0.001$ \\
\hline & No & $2863(18)$ & $2.38(1.2)$ & & $2.92(1.1)$ & \\
\hline & Mon-Fri daytime hours only & $12749(80)$ & $2.39(1.2)$ & $<0.001$ & $3.19(1.1)$ & 0.005 \\
\hline & Also evening/weekend shifts & $1937(12)$ & $2.15(1.1)$ & & $3.19(1.0)$ & \\
\hline & Also night shifts & $1195(8)$ & $1.69(1.0)$ & & $3.09(1.0)$ & \\
\hline \multirow{2}{*}{ Smoking } & No & $12990(83)$ & $2.31(1.2)$ & 0.313 & $3.16(1.0)$ & $<0.001$ \\
\hline & Yes & $2696(17)$ & $2.28(1.2)$ & & $3.25(1.0)$ & \\
\hline \multirow{2}{*}{$\begin{array}{l}\text { Consumption of } \\
\text { alcohol }\end{array}$} & Low, $\leqslant 200 \mathrm{~g} /$ week & $12564(88)$ & $2.31(1.2)$ & $<0.001$ & $3.18(1.0)$ & 0.079 \\
\hline & High, $>200 \mathrm{~g} /$ week & $1696(12)$ & $2.42(1.3)$ & & $3.23(1.1)$ & \\
\hline \multirow[t]{2}{*}{ Overweight } & No, $B M I \leqslant 27$ & $12030(76)$ & $2.30(1.2)$ & 0.375 & $3.16(1.0)$ & $<0.001$ \\
\hline & Yes, $\mathrm{BMI}>27$ & $3799(24)$ & $2.32(1.2)$ & & $3.24(1.0)$ & \\
\hline \multirow{2}{*}{$\begin{array}{l}\text { Sedentary } \\
\text { lifestyle }\end{array}$} & No, $\geqslant 30$ min fast walking/week & $13761(86)$ & $2.31(1.2)$ & 0.906 & $3.18(1.0)$ & 0.138 \\
\hline & Yes, $<30 \mathrm{~min}$ & $2211(14)$ & $2.30(1.3)$ & & $3.15(1.1)$ & \\
\hline \multirow[t]{4}{*}{ Job control } & 4 high & $4538(28)$ & $2.67(1.2)$ & $<0.001$ & $3.28(1.1)$ & $<0.001$ \\
\hline & 3 & $3797(24)$ & $2.38(1.2)$ & & $3.20(1.0)$ & \\
\hline & 2 & $4173(26)$ & $2.17(1.1)$ & & $3.16(1.0)$ & \\
\hline & 1 low & 3529 (22) & $1.91(1.0)$ & & $3.04(1.0)$ & \\
\hline \multirow[t]{4}{*}{ Job demands } & 1 low & $3911(24)$ & $2.29(1.2)$ & 0.427 & $3.37(1.0)$ & $<0.001$ \\
\hline & 2 & 4318 (27) & $2.29(1.2)$ & & $3.21(1.0)$ & \\
\hline & 3 & $4182(26)$ & $2.32(1.2)$ & & $3.10(1.0)$ & \\
\hline & 4 high & $3617(23)$ & $2.33(1.2)$ & & $3.04(1.1)$ & \\
\hline \multirow{4}{*}{$\begin{array}{l}\text { Job strain } \\
\text { situations }\end{array}$} & low strain & $4113(26)$ & $2.47(1.2)$ & $<0.001$ & $3.31(1.1)$ & $<0.001$ \\
\hline & active job & $4210(26)$ & $2.60(1.2)$ & & 3.18 (1.1) & \\
\hline & passive job & 4107 (26) & 2.11 (1.1) & & $3.26(0.9)$ & \\
\hline & high strain & $3582(22)$ & $1.99(1.1)$ & & $2.94(1.0)$ & \\
\hline \multirow{4}{*}{$\begin{array}{l}\text { Effort-reward } \\
\text { imbalance }\end{array}$} & 1 low & 3046 (19) & $2.46(1.2)$ & $<0.001$ & $3.29(1.0)$ & $<0.001$ \\
\hline & 2 & $4783(30)$ & $2.41(1.2)$ & & $3.26(1.0)$ & \\
\hline & 3 & 4888 (31) & 2.25 (1.2) & & 3.15 (1.0) & \\
\hline & 4 high & 3283 (21) & $2.10(1.2)$ & & $3.00(1.1)$ & \\
\hline
\end{tabular}

worktime control scores according to work units. Each respondent's work unit was identified from the employers' records based on a five level organisational hierarchy classification. For each level, we calculated mean control over daily working hours and control over days off. The resulting aggregate scores of the lowest level-that is, work unit-were assigned to each member of the unit. If the number of participants in the unit was nine or less, we used values aggregated onto the next level. Thus, in all cases, the aggregated scores for worktime control were based on values derived from 10 or more individual respondents. There were 792 categories of control over daily working hours and 789 categories of control over days off.

All analyses, stratified for gender, were adjusted for demographics (age, level of education, occupational status, work contract, schedule type, marital status, and presence of dependent children) and behavioural and biological health risks (smoking, alcohol consumption, sedentary lifestyle, and overweight).

The SAS program package was used to perform all analyses. Poisson regression models were calculated using the GENMOD procedure.

\section{RESULTS}

Table 1 shows the participants' descriptive statistics. Men reported higher control over their daily working hours than did women. Control over days off did not vary by gender. All background variables except marital status and sedentary lifestyle were associated with worktime control. Low job control was associated with poor worktime control of both types, and high job demands with poor control over days off. The respondents in high strain jobs and with high effortreward imbalance reported lower worktime control than those in other job situations. 
Table 2 Main effects: adjusted* rate ratios (95\% confidence intervals) of medically certified sickness absences by levels of worktime control and sources of stress

\begin{tabular}{|c|c|c|c|c|c|}
\hline & & Number(\%) & Women & Number(\%) & Men \\
\hline Control over & 4 good & 2364 (19) & 1.00 & $1130(28)$ & 1.00 \\
\hline daily & 3 & 3085 (25) & $1.16(1.09,1.23)$ & $827(21)$ & $1.05(0.93,1.18)$ \\
\hline working & 2 & 3261 (27) & $1.26(1.18,1.33)$ & 757 (19) & $1.18(1.05,1.33)$ \\
\hline hours & 1 poor & 3417 (28) & $1.29(1.22,1.37)$ & 1298 (32) & $1.26(1.13,1.40)$ \\
\hline Control over & 4 good & 1399 (12) & 1.00 & $512(13)$ & 1.00 \\
\hline \multirow[t]{3}{*}{ days off } & 3 & 4477 (37) & $1.05(0.98,1.12)$ & 1504 (37) & $1.19(1.05,1.35)$ \\
\hline & 2 & 3699 (31) & $1.16(1.09,1.24)$ & 1164 (29) & $1.20(1.05,1.36)$ \\
\hline & 1 poor & 2552 (21) & $1.23(1.14,1.32)$ & $832(21)$ & $1.26(1.09,1.45)$ \\
\hline \multirow[t]{4}{*}{ Job demands } & 1 low & 2860 (24) & 1.00 & $1051(26)$ & 1.00 \\
\hline & 2 & $3164(26)$ & $1.09(1.03,1.15)$ & 1175 (29) & $1.10(1.00,1.22)$ \\
\hline & 3 & $3158(26)$ & $1.13(1.06,1.19)$ & $1024(26)$ & $1.18(1.07,1.31)$ \\
\hline & 4 high & $2880(24)$ & $1.26(1.19,1.33)$ & 737 (18) & $1.16(1.03,1.30)$ \\
\hline \multirow{4}{*}{ Job control } & 4 high & $3433(28)$ & 1.00 & 1105 (28) & 1.00 \\
\hline & 3 & $2830(23)$ & $0.96(0.91,1.01)$ & $967(24)$ & $0.98(0.87,1.09)$ \\
\hline & 2 & $3129(26)$ & $1.11(1.05,1.17)$ & $1053(26)$ & $0.98(0.88,1.09)$ \\
\hline & 1 low & 2667 (22) & $1.09(1.03,1.15)$ & $862(22)$ & $1.17(1.05,1.32)$ \\
\hline \multirow[t]{4}{*}{ Job strain } & Low strain & 3054 (25) & 1.00 & 1059 (27) & 1.00 \\
\hline & Active job & 3199 (27) & $1.09(1.03,1.15)$ & 1011 (25) & $1.04(0.93,1.17)$ \\
\hline & Passive job & 2942 (24) & $1.07(1.01,1.13)$ & 1165 (29) & $1.03(0.93,1.14)$ \\
\hline & High strain & 2832 (24) & $1.27(1.20,1.34)$ & 750 (19) & $1.21(1.08,1.35)$ \\
\hline Effort- & 1 low & 2124 (18) & 1.00 & $922(23)$ & 1.00 \\
\hline reward & 2 & 3601 (30) & $0.99(0.94,1.05)$ & $1182(30)$ & $1.10(0.98,1.22)$ \\
\hline \multirow[t]{2}{*}{ imbalance } & 3 & 3731 (31) & $1.03(0.98,1.10)$ & 1157 (29) & $1.10(0.99,1.23)$ \\
\hline & 4 high & $2563(21)$ & $1.21(1.14,1.29)$ & 729 (18) & $1.41(1.26,1.58)$ \\
\hline
\end{tabular}

*Adjusted for age, level of education, occupational status, types of work contract and work schedule, marital status and dependent children, smoking, alcohol consumption, sedentary lifestyle, and overweight.

Table 3 Combined effects of control over daily working hours with sources of stress: adjusted* rate ratios $195 \%$ confidence intervals) for medically certified sickness absences by level of control over daily working hours combined with level of job demands, job control, job strain, and effort-reward imbalance

\begin{tabular}{|c|c|c|c|c|c|c|c|}
\hline & & \multicolumn{2}{|c|}{ Control over daily working hours } & \multirow{3}{*}{$\begin{array}{l}\text { p for inter- } \\
\text { action }\end{array}$} & \multicolumn{2}{|c|}{ Control over daily working hours } & \multirow{3}{*}{$\begin{array}{l}\mathrm{p} \text { for inter } \\
\text { action }\end{array}$} \\
\hline & & \multicolumn{2}{|c|}{ Individual measures } & & \multicolumn{2}{|c|}{ Workplace aggregates } & \\
\hline & & good & poor & & good & poor & \\
\hline \multicolumn{8}{|l|}{ Women } \\
\hline \multirow{3}{*}{ demands } & 1 low & 1.00 & $1.06(0.97,1.14)$ & 0.017 & 1.00 & $1.15(1.06,1.25)$ & 0.189 \\
\hline & 3 & $1.02(0.93,1.10)$ & $1.27(1.18,1.37)$ & & $1.14(1.06,1.23)$ & $1.25(1.15,1.35)$ & \\
\hline & 4 high & $1.16(1.07,1.26)$ & $1.40(1.30,1.51)$ & & $1.24(1.15,1.33)$ & $1.45(1.35,1.57)$ & \\
\hline & 4 high & $1.00^{\circ}$ & $1.05(0.98,1.13)$ & $<0.001$ & 1.00 & $1.07(0.99,1.16)$ & 0.042 \\
\hline \multirow[t]{3}{*}{ control } & 3 & $0.96(0.89,1.04)$ & $1.00(0.93,1.08)$ & & $0.93(0.86,1.00)$ & $1.08(1.00,1.17)$ & \\
\hline & 2 & $0.99(0.91,1.07)$ & $1.25(1.16,1.34)$ & & $1.05(0.98,1.13)$ & $1.28(1.19,1.37)$ & \\
\hline & 1 low & $0.93(0.85,1.02)$ & $1.23(1.15,1.33)$ & & $1.02(0.95,1.10)$ & $1.27(1.18,1.37)$ & \\
\hline \multirow{4}{*}{$\begin{array}{l}\text { Job } \\
\text { strain }\end{array}$} & Low strain & 1.00 & $0.96(0.89,1.04)$ & $<0.001$ & 1.00 & $1.04(0.96,1.13)$ & $<0.001$ \\
\hline & Active job & $1.00(0.93,1.08)$ & $1.13(1.05,1.23)$ & & $1.03(0.96,1.10)$ & $1.22(1.12,1.31)$ & \\
\hline & Passive job & $0.92(0.85,1.00)$ & $1.15(1.07,1.24)$ & & $0.95(0.89,1.03)$ & $1.30(1.20,1.40)$ & \\
\hline & High strain & $1.05(0.96,1.14)$ & $1.36(1.26,1.46)$ & & $1.24(1.16,1.34)$ & $1.35(1.26,1.46)$ & \\
\hline \multirow{4}{*}{$\begin{array}{l}\text { Effort- } \\
\text { reward } \\
\text { imbalance }\end{array}$} & 1 low & 1.00 & $1.18(1.08,1.30)$ & 0.856 & 1.00 & $1.18(1.08,1.30)$ & 0.114 \\
\hline & 2 & $1.00(0.92,1.09)$ & $1.15(1.06,1.25)$ & & $0.97(0.90,1.05)$ & $1.19(1.10,1.29)$ & \\
\hline & 3 & $1.03(0.94,1.12)$ & $1.20(1.11,1.30)$ & & $1.03(0.95,1.11)$ & $1.21(1.21,1.31)$ & \\
\hline & 4 high & $1.23(1.12,1.35)$ & $1.39(1.27,1.51)$ & & $1.26(1.16,1.36)$ & $1.36(1.25,1.48)$ & \\
\hline \multicolumn{8}{|c|}{ 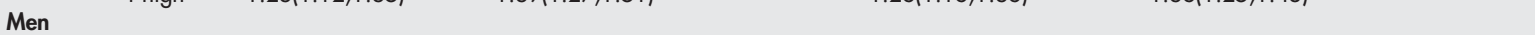 } \\
\hline & 1 low & 1.00 & $1.07(0.92,1.24)$ & 0.144 & 1.00 & $1.07(0.93,1.24)$ & 0.609 \\
\hline \multirow[t]{3}{*}{ demands } & & $1.01(0.86,1.19)$ & $1.23(1.06,1.42)$ & & $1.14(1.00,1.31)$ & $1.14(0.99,1.31)$ & \\
\hline & 3 & $1.08(0.92,1.28)$ & $1.32(1.13,1.53)$ & & $1.15(1.00,1.32)$ & $1.30(1.12,1.50)$ & \\
\hline & 4 high & $0.98(0.81,1.17)$ & $1.37(1.16,1.62)$ & & $1.18(1.01,1.38)$ & $1.20(1.02,1.43)$ & \\
\hline \multirow{4}{*}{ control } & 4 high & 1.00 & $1.27(1.09,1.48)$ & 0.484 & 1.00 & $1.08(0.91,1.27)$ & 0.088 \\
\hline & 3 & $1.00(0.86,1.17)$ & $1.18(1.01,1.37)$ & & $1.04(0.91,1.19)$ & $0.92(0.78,1.10)$ & \\
\hline & 2 & $0.97(0.83,1.14)$ & $1.18(1.02,1.36)$ & & $0.92(0.81,1.07)$ & $1.10(0.95,1.27)$ & \\
\hline & 1 low & $1.28(1.06,1.54)$ & $1.36(1.18,1.57)$ & & $1.20(1.03,1.40)$ & $1.22(1.06,1.40)$ & \\
\hline Job & Low strain & 1.00 & $0.98(0.84,1.14)$ & $<0.001$ & 1.00 & $0.88(0.75,1.05)$ & 0.127 \\
\hline \multirow{3}{*}{ strain } & Active job & $0.85(0.73,1.00)$ & $1.29(1.11,1.51)$ & & $0.98(0.86,1.12)$ & $1.04(0.88 .1 .24)$ & \\
\hline & Passive job & $0.85(0.72,1.01)$ & $1.11(0.97,1.28)$ & & $0.92(0.81,1.06)$ & $1.05(0.92,1.20)$ & \\
\hline & High strain & $1.20(1.01,1.43)$ & $1.21(1.05,1.41)$ & & $1.14(0.97,1.32)$ & $1.19(1.03,1.38)$ & \\
\hline Effort- & 1 low & 1.00 & $1.01(0.86,1.20)$ & 0.330 & 1.00 & $1.03(0.87,1.22)$ & 0.939 \\
\hline reward & 2 & $1.00(0.85,1.18)$ & $1.18(1.01,1.38)$ & & $1.07(0.93,1.24)$ & $1.15(0.99,1.34)$ & \\
\hline \multirow{2}{*}{ imbalance } & 3 & $0.99(0.84,1.16)$ & $1.21(1.04,1.41)$ & & $1.11(0.97,1.28)$ & $1.12(0.97,1.31)$ & \\
\hline & 4 high & $1.24(1.03,1.50)$ & $1.51(1.29,1.77)$ & & $1.40(1.19,1.63)$ & $1.46(1.25,1.70)$ & \\
\hline
\end{tabular}


Table 4 Combined effects of control over days off with sources of stress: adjusted ${ }^{*}$ rate ratios (95\% confidence intervals) for medically certified sickness absences by level of control over days off combined with level of job demands, job control, job strain, and effort-reward imbalance

\begin{tabular}{|c|c|c|c|c|c|c|c|}
\hline & & \multicolumn{2}{|c|}{ Control over days off } & \multirow{2}{*}{$p$ for inter } & \multicolumn{2}{|c|}{ Control over days off } & \multirow{3}{*}{$\begin{array}{l}\text { p for inter- } \\
\text { action }\end{array}$} \\
\hline & & \multicolumn{2}{|c|}{ Individual measures } & & \multicolumn{2}{|c|}{ Workplace aggregates } & \\
\hline & & good & poor & action & good & poor & \\
\hline \multirow{4}{*}{$\begin{array}{l}\text { Women } \\
\text { Job } \\
\text { demands }\end{array}$} & 1 low & 1.00 & $1.06(0.98,1.16)$ & \multirow{4}{*}{0.131} & 1.00 & $1.11(1.02,1.20)$ & \multirow{4}{*}{0.353} \\
\hline & 2 & $1.05(0.98,1.13)$ & $1.19(1.10,1.28)$ & & $1.05(0.98,1.14)$ & $1.23(1.14,1.33)$ & \\
\hline & 3 & $1.04(0.96,1.12)$ & $1.25(1.16,1.34)$ & & $1.12(1.04,1.21)$ & $1.22(1.13,1.32)$ & \\
\hline & 4 high & $1.24(1.15,1.34)$ & $1.34(1.25,1.44)$ & & $1.28(1.19,1.38)$ & $1.36(1.26,1.46)$ & \\
\hline Job & 4 high & 1.00 & $1.11(1.03,1.19)$ & \multirow[t]{4}{*}{0.003} & 1.00 & $1.06(0.98,1.14)$ & \multirow[t]{4}{*}{$<0.001$} \\
\hline \multirow[t]{3}{*}{ control } & 3 & $1.00(0.93,1.08)$ & $1.00(0.92,1.08)$ & & $0.97(0.90,1.05)$ & $1.00(0.93,1.08)$ & \\
\hline & 2 & $1.06(0.98,1.14)$ & $1.25(1.16,1.34)$ & & $1.09(1.01,1.17)$ & $1.19(1.11,1.28)$ & \\
\hline & 1 low & $1.02(0.94,1.10)$ & $1.23(1.14,1.33)$ & & $1.00(0.92,1.08)$ & $1.27(1.17,1.37)$ & \\
\hline Job & Low strain & 1.00 & $1.03(0.95,1.11)$ & \multirow[t]{4}{*}{0.037} & 1.00 & $1.07(0.99,1.16)$ & \multirow[t]{4}{*}{0.029} \\
\hline \multirow{3}{*}{ strain } & Active job & $1.06(0.99,1.15)$ & $1.13(1.05,1.22)$ & & $1.11(1.02,1.20)$ & $1.14(1.05,1.23)$ & \\
\hline & Passive job & $1.01(0.94,1.09)$ & $1.17(1.08,1.26)$ & & $1.02(0.95,1.10)$ & $1.23(1.13,1.33)$ & \\
\hline & High strain & $1.15(1.06,1.25)$ & $1.36(1.27,1.46)$ & & $1.23(1.14,1.33)$ & $1.37(1.28,1.48)$ & \\
\hline \multirow{4}{*}{$\begin{array}{l}\text { Effort- } \\
\text { reward } \\
\text { imbalance }\end{array}$} & 1 low & 1.00 & $1.15(1.05,1.27)$ & \multirow[t]{4}{*}{$<0.001$} & 1.00 & $1.08(0.99,1.19)$ & \multirow[t]{4}{*}{0.251} \\
\hline & 2 & $1.03(0.95,1.12)$ & $1.08(1.00,1.18)$ & & $0.95(0.88,1.04)$ & $1.12(1.03,1.21)$ & \\
\hline & 3 & $1.07(0.99,1.16)$ & $1.13(1.04,1.22)$ & & $1.01(0.93,1.10)$ & $1.14(1.05,1.24)$ & \\
\hline & 4 high & $1.12(1.02,1.22)$ & $1.43(1.32,1.55)$ & & $1.23(1.13,1.33)$ & $1.30(1.19,1.41)$ & \\
\hline \multirow{5}{*}{$\begin{array}{l}\text { Men } \\
\text { Job } \\
\text { demands }\end{array}$} & & & & \multirow{5}{*}{0.019} & & & \\
\hline & 1 low & 1.00 & $1.06(0.91,1.23)$ & & 1.00 & $0.87(0.75,1.01)$ & \multirow{4}{*}{0.230} \\
\hline & 2 & $1.14(1.00,1.30)$ & $1.12(0.98,1.28)$ & & $1.09(0.95,1.25)$ & $0.98(0.85,1.12)$ & \\
\hline & 3 & $1.06(0.91,1.23)$ & $1.34(1.17,1.54)$ & & $1.08(0.93,1.26)$ & $1.12(0.97,1.28)$ & \\
\hline & 4 high & $1.24(1.06,1.46)$ & $1.13(0.96,1.32)$ & & $1.05(0.89,1.25)$ & $1.10(0.94,1.28)$ & \\
\hline Job & 4 high & 1.00 & $1.15(0.98,1.34)$ & \multirow[t]{4}{*}{0.366} & 1.00 & $1.03(0.88,1.21)$ & \multirow[t]{4}{*}{0.212} \\
\hline \multirow[t]{3}{*}{ control } & 3 & $1.02(0.88,1.19)$ & $1.05(0.89,1.23)$ & & $1.09(0.93,1.28)$ & $0.90(0.76,1.07)$ & \\
\hline & 2 & $1.06(0.92,1.23)$ & $1.01(0.87,1.17)$ & & $1.05(0.90,1.22)$ & $0.96(0.82,1.12)$ & \\
\hline & 1 low & $1.19(1.02,1.39)$ & $1.28(1.11,1.47)$ & & $1.21(1.03,1.42)$ & $1.20(1.03,1.39)$ & \\
\hline Job & Low strain & 1.00 & $0.98(0.83,1.15)$ & \multirow[t]{4}{*}{0.115} & 1.00 & $0.87(0.75,1.02)$ & \multirow[t]{4}{*}{0.263} \\
\hline \multirow[t]{3}{*}{ strain } & Active job & $0.94(0.81,1.09)$ & $1.15(0.99,1.35)$ & & $0.97(0.82,1.14)$ & $0.97(0.84,1.14)$ & \\
\hline & Passive job & $0.99(0.86,1.13)$ & $1.04(0.91,1.19)$ & & $1.02(0.83,1.17)$ & $0.91(0.79,1.05)$ & \\
\hline & High strain & $1.23(1.05,1.44)$ & $1.17(1.02,1.35)$ & & $1.10(0.93,1.30)$ & $1.16(1.00,1.34)$ & \\
\hline Effort- & 1 low & 1.00 & $0.97(0.82,1.15)$ & 0.051 & 1.00 & $1.06(0.89,1.25)$ & 0.154 \\
\hline reward & 2 & $0.98(0.84,1.13)$ & $1.20(1.04,1.40)$ & & $1.12(0.95,1.31)$ & $1.13(0.97,1.32)$ & \\
\hline imbalance & 3 & $1.10(0.95,1.28)$ & $1.07(0.92,1.24)$ & & $1.22(1.05,1.43)$ & $1.05(0.90,1.23)$ & \\
\hline & 4 high & $1.39(1.18,1.64)$ & $1.38(1.18,1.61)$ & & $1.57(1.33,1.85)$ & $1.37(1.17,1.61)$ & \\
\hline
\end{tabular}

*Adjusted for age, level of education, occupational status, types of work contract and work schedule, marital status and dependent children, smoking, alcohol consumption, sedentary lifestyle, and overweight.

Worktime control and job stress predicted sickness absences among both sexes (table 2). The employees in the poorest quartiles of control over daily working hours and days off had 1.2-1.3 times higher rates of subsequent sickness absences than those in the best quartiles. Compared with those in low strain jobs, the women and the men in high-strain jobs had 1.2-1.3 times higher rates of subsequent sickness absences. The women within the highest quartile of effort-reward imbalance had 1.2 times more sickness absences than those with least imbalance, and the corresponding figure for the men was 1.4.

\section{Combined effects of control over daily working hours with sources of work stress}

Table 3 shows the joint effects of work stress with control over daily working hours, as indicated by individual and aggregated scores, on sickness absence. Among the women, high demands, low control, and high strain were in a

\section{What is already known on this topic}

- Work stress (job strain, effort-reward imbalance) has widespread negative consequences on public health.

- Low worktime control has been shown to increase the risks of subjective ill health and sickness absence, especially among women. stepwise manner associated with higher rates of sickness absence, mainly in combination with poor control over daily working hours. Compared with women having both low stress and good control over their daily working hours, women in high stress jobs had 1.2-1.4 times higher rates of sickness absence if they simultaneously had poor control over their working hours, but no or only a slight increase in absence rate if their control over working hours was good. The analyses using the aggregated measure of control over working hours largely replicated these results. Significant interactions of job demands, job control, and job strain with control over working hours gave additional statistical support to these joint effects.

The findings were less consistent among the men. High levels of job demands and effort-reward imbalance predicted higher rates of sickness absences mainly in combination with poor control over working hours, when measured individually. Interestingly, poor control over working hours also predicted increased sickness absence among the men who had high job control and worked in an active job. However,

\section{What this paper adds}

Female employees, and to a lesser extent, male employees, exposed to work stress maintained lowered rates of sickness absence if they had good control over their working times. 


\section{Policy implication}

Providing employees with worktime control may promote health in strenuous work life.

none of these results were replicated in analyses using the aggregated measure of control over daily working hours.

\section{Combined effects of control over days off with sources of work stress}

Table 4 shows the joint effects of work stress and control over days off on sickness absence. Among the women, low job control, high strain and effort-reward imbalance predicted high rates of sickness absence in combination with poor control over days off, but the associations were absent or weak if control over days off was good. Among the men, the effects of work stress on sickness absence were not dependent on control over days off.

We also studied the combined effects of control over daily working hours and days off with decision authority, a subcomponent of job control (data not shown). These results were similar to those obtained with job control.

\section{DISCUSSION}

In this large cohort of public sector employees, work stress and low worktime control predicted increased rates of medically certified sickness absence. Importantly, the adverse effects of work stress were reduced by good employee control over daily working hours and days off, especially among the women. High job demands, low control, high strain, and high effort-reward imbalance predicted health problems more strongly for the women with poor worktime control than for those with good worktime control. These findings were evident using both individual and aggregated measures of worktime control. Among the men, the effects of high demands and high effort-reward imbalance on sickness absence were smaller in combination with good control over daily working hours, measured individually, but not with more objective workplace aggregates. To our knowledge, this is the first longitudinal study to examine the role of worktime control against health problems among employees loaded with work stress.

Our findings suggest that worktime control may to some extent help in coping with important sources of work stress, such as job strain and effort-reward imbalance. Capturing a specific control dimension, worktime control may reduce stress by enhancing perceived work related autonomy, especially when an employee has otherwise little control over work $^{18}{ }^{29}$ or is in an unsatisfying work situation. In practical terms, worktime control may enable working at times of best possible resources and support, and thus decrease accumulation of particularly stressful work situations.

The finding that worktime control moderates the health effects of work stress could have practical implications for actions aiming to prevent stress related illness in the work life. Previously, job strain and effort-reward imbalance have been detected among the strongest psychosocial predictors of work related ill health, ${ }^{41-13}$ indicating the need for in-depth improvements in working conditions. Meanwhile, these findings suggest that the negative consequences of work stress are lowered in combination with good control over working times. These results, however, do not indicate that worktime control alone would be the answer to tackling work stress, as flexibility may associate with increased demands at work. $^{30}$ There is already a trend in all Western countries towards increasing diversity and flexibility of working times with so far poorly known effects on health. ${ }^{30}{ }^{31}$ In studying these effects, a distinction between company based flexibility and individual oriented flexibility, the focus of our study, is important. ${ }^{3}$

Methodologically, the strengths of this study include its prospective design capturing an effect in a large cohort covering a variety of occupations, the control for several potential confounders, and the replication of the findings using both individual and aggregated measures of worktime control. By using routinely collected sickness absence records we could further eliminate the subjectivity problem. Baseline health status was controlled for, as before the survey the respondents had no health problems severe enough to lead to a sick leave certified by a doctor, and were thus likely to be initially healthy. Medically certified sickness absence is a valid measure of health in working populations, a strong predictor of early retirement and mortality in epidemiological studies. ${ }^{20-22}$

The limitations of the study include a crude measure of effort-reward imbalance, not assessing over-commitment (see Siegrist et al), ${ }^{32}$ and the reliance on only one health indicator. Our sample is restricted to full time public sector employees with permanent or longish work contracts. To evaluate the generalisability of the findings, further research is needed among private sector workers and among populations with a more even gender distribution and more manual and shift workers as well as among part time and temporary employees. Finally, the role of worktime control in preventing stress related health problems should be examined in properly conducted intervention studies.

To conclude, female employees, and to a lesser extent, male employees, exposed to work stress maintained lowered rates of sickness absence if they had good control over their working times.

\section{Authors' affiliations}

L Ala-Mursula, Centre of Occupational Health, City of Oulu, and Department of Public Health Science and General Practice, University of Oulu, Finland

J Vahtera, A Linna, J Pentti, Finnish Institute of Occupational Health, Turku, Finland

M Kivimäki, Department of Psychology, University of Helsinki, Helsinki, Finland

Funding: this study was supported by the Academy of Finland (project 105195, grant 106645), the Finnish Work Environment Fund (projects 101190 and 103432) and the participating towns. The sponsors of the study had no role in the design and conduct of the study; collection, management, analysis, and interpretation of the data; and preparation, review, or approval of the manuscript.

Competing interests: none.

\section{REFERENCES}

1 Stansfeld SA, Bosma $\mathrm{H}$, Hemingway $\mathrm{H}$, et al. Psychosocial work characteristics and social support as predictors of SF-36 health functioning: the Whitehall II study. Psychosom Med 1998;60:247-55.

2 Amick BC III, Kawachi I, Coakley EH, et al. Relationship of job strain and isostrain to health status in a cohort of women in the United States. Scand J Work Environ Health 1998;24:54-61.

3 Stansfeld SA, Fuhrer R, Shipley MJ, et al. Work characteristics predict psychiatric disorder: prospective results from the Whitehall II study. Occup Environ Med 1999;56:302-7.

4 Bosma H, Peter R, Siegrist J, et al. Two alternative job stress models and the risk of coronary heart disease. Am J Public Health 1998;88:68-74

5 Kuper H, Singh-Manoux A, Siegrist J, et al. When reciprocity fails: effortreward imbalance in relation to coronary heart disease and health functioning within the Whitehall II study. Occup Environ Med 2002;59:777-84.

6 North F, Syme SL, Feeney A, et al. Psychosocial work environment and sickness absence among British civil servants: the Whitehall II study. Am J Public Health 1996:86:332-40.

7 Niedhammer I, Bugel I, Goldberg M. Psychosocial factors at work and sickness absence in the Gazel cohort: a prospective study. Occup Environ Med 1998;55:735-41 
8 Vahtera J, Kivimäki M, Pentti J, et al. Effect of change in the psychosocial work environment on sickness absence: a seven year follow up of initially healthy employees. J Epidemiol Community Health 2000;54:484-93.

9 Johnson JV, Stewart W, Hall EM, et al. Long-term psychosocial work environment and cardiovascular mortality among Swedish men. Am J Public Health 1996;86:324-31.

10 Amick BC III, McDonough P, Chang $\mathrm{H}$, et al. Relationships between all-cause mortality and cumulative working life course psychosocial and physical exposures in the United States labor market from 1968 to 1992. Psychosom Med 2002;64:370-81.

11 Kivimäki M, Leino-Arjas P, Luukkonen R, et al. Work stress and risk of cardiovascular mortality: prospective cohort study of industrial employees. BMJ.com 2002;325:857.

12 Karasek R, Theorell T. Healthy work: stress, productivity, and the reconstruction of working life. New York: Basic Books, 1990.

13 Siegrist J. Adverse health effects of high-effort/low-reward conditions. J Occup Health Psychol 1996;1:27-41.

14 European Commission, Employment and social affairs. Guidance on workrelated stress. Spice of life or kiss of death? Executive summary. Luxembourg: Office for Official Publications of the European Communities, 2002:9-11

15 Ala-Mursula L, Vahtera J, Kivimäki M, et al. Employee control over working times: associations with subjective health and sickness absences. J Epidemiol Community Health 2002;56:272-8.

16 Ala-Mursula L, Vahtera J, Pentti J, et al. Effect of employee worktime control on health: a prospective cohort study. Occup Environ Med 2004;61:254-61

17 Fagan C, Burchell B. Gender, jobs and working conditions in the European Union. European Foundation for the Improvement of Living and Working Conditions. Luxembourg: Office for Official Publications of the European Communities, 2002:57-79.

18 Fenwick R, Tausig M. Scheduling Stress. Family and health outcomes of shift work and schedule control. American Behavioral Scientist 2001:44:1179-98.

19 Hammar N, Alfredsson L, Theorell T. Job characteristics and the incidence of myocardial infarction. Int J Epidemiol 1994;23:277-84.
20 Kivimäki M, Head J, Ferrie JE, et al. Sickness absence as a global measure of health: evidence from all-cause mortality in the Whitehall II study. BMJ 2003;327:364-9

21 Vahtera J, Pentti J, Kivimäki M. Sickness absence as a predictor of mortality among male and female employees. J Epidemiol Community Health 2004;58:321-6.

22 Kivimäki M, Forma P, Wikström J, et al. Sickness absence as a risk marker of future disability pension: the 10 town study. J Epidemiol Community Health 2004;58:710-11.

23 Karasek RA. Job content questionnaire and user's guide. Revision 1.1. Lowell, MA: University of Massachusetts, Department of Work Environment, 1985.

24 Taris TW, Kalimo R, Schaufeli WB. Inequity at work: its measurement and association with worker health. Work Stress 2002;16:287-301.

25 Kouvonen A, Kivimäki M, Virtanen $M$, et al. Work stress, smoking status, and smoking intensity: an observational study of 46190 employees J Epidemiol Community Health 2005;59:63-9.

26 Virtanen $M$, Kivimäki $M$, Elovainio $M$, et al. From insecure to secure employment: changes in work, health, health related behaviours, and sickness absence. Occup Environ Med 2003:60:948-53.

27 Statistics Finland. Classification of occupations, handbook number 14. Helsinki: Statistics Finland, 1997.

28 Knauth $\mathrm{P}$, Hornberger S. Preventive and compensatory measures for shiff workers. Occup Med (Oxf) 2003;53:109-16.

29 Baltes B, Briggs TE, Huff JW, et al. Flexible and compressed workweek schedules: a meta-analysis of their effects on work-related criteria. J Appl Psychol 1999;84:496-513.

30 Naegele G, Barkholdt C, deVroom J, et al. A new organisation of time over working life. European Foundation for the Improvement of Living and Working Conditions. Luxembourg: Office for Official Publications of the European Communities, 2003:55-9.

31 Costa G, Åkerstedt T, Nachreiner F, et al. Flexible work hours, health and well being: results of the SALTSA project. Shiftwork International Newsletter 2003;20:55.

32 Siegrist J, Starke D, Chandola T, et al. The measurement of effort-reward imbalance at work: European comparisons. Soc Sci Med 2004;58:1483-99. 